\title{
A critical assessment of the consciousness by synchrony hypothesis
}

\author{
ANDRÉS F. CANALES ${ }^{1}$, DAVID M. GÓMEZ ${ }^{2}$ and CLAUDIO R. MAFFET ${ }^{3}$
}

${ }^{1}$ Facultad de Ciencias, Universidad de Valparaíso, andres.canales.j@gmail.com
2 Departamento de Ingeniería Matemática, Universidad de Chile,dgomez@dim.uchile.cl
${ }^{3}$ Departamento de Anatomía, Universidad de Viña del Mar, claudio.maffet@gmail.com

\section{INTRODUCTION}

The understanding of consciousness has been one of the biggest challenges for cognitive science (e.g. Cosmelli et al., 2007; Crick and Koch, 2003; Seth et al., 2006), and the study of the visual system has been its theoretical and empirical starting point. For instance, the consciousness by synchrony hypothesis postulates neuronal synchronization as the putative mechanism of visual consciousness. In this article, we criticize this idea and the traditional arguments supporting it. First, we present the relation between the consciousness by synchrony hypothesis and the visual binding problem. Then, based on empirical evidence, we analyze the limitations of neuronal synchrony to solve both this specific problem and the more general visual consciousness issue. We end by considering a number of additional problems for the consciousness by synchrony hypothesis.

\section{NEURONAL SYNCHRONY, BINDING AND CONSCIOUSNESS}

The problem of combining different visual features into unitary coherent visual percepts is known as the "binding problem" (e.g. see Roskies, 1999). In the eighties, the existence of precise temporal correlations between neuronal discharges was postulated as a solution to this binding problem (e.g. von der Malsburg, 1981; Gray, 1999). This theory holds that "neurons that respond to features of one object fire their action potentials at the same time, but neurons responding to features of different objects do not" (Roelfsema, 2004, p. 982). Recently, Engel and Singer (2001) have proposed that some facets of the binding problem also apply to the issue of consciousness. This would be pertinent because "unraveling the mechanism capable of solving the binding problem may be critical for understanding the NCC (i.e. neural correlates of consciousness)" (p. 17). Thus, neuronal synchronization has been claimed as a plausible mechanism that would participate in the emergence of visual consciousness (Engel et al. 1999). Moreover, the consciousness by synchrony hypothesis pushes this assumption further holding that neuronal synchrony would be the putative mechanism of visual awareness (Crick and Koch, 1990; Singer, 2001). We argue, based on empirical evidence, that neuronal synchrony is incapable of explaining this phenomenon.

\section{LIMITATIONS OF NEURONAL SYNCHRONY IN VISUAL BINDING}

In previous critical reviews, several limitations have been presented for the role of neuronal synchrony in solving the binding problem (Movshon and Shadlen, 1999). For instance, for most of the empirical studies supporting the role of neuronal synchrony in visual grouping the perceptual status of their observer animals is unknown (e.g. Gray et al., 1989; Engel et al, 1991; Kreiter and Singer, 1996). Similar limitations apply to the majority of the studies against the role of neuronal synchrony in contour grouping (e.g Thiele and Stoner, 2003; Palanca and DeAngelis, 2005). To solve the so-called binding 
problem, neuronal synchrony would have to provide the means to bind together all the features of one object and segregate them from features of other objects and the background. In order to clarify this, Roelfsema et al., (2004) conducted a contour grouping study where monkeys had to group together all contour segments belonging to a target curve. One of the crucial advantages of this study is that "monkeys report about perceptual grouping on every trial, and we can therefore be confident about their perceptual organization" (ibid. p.982). The authors recorded differences in synchrony between responses evoked by grouped contours and those evoked by non-grouped contours in primary visual area V1. It was found that synchronization was unrelated to the grouping of contours into elongated curves. This evidence strongly suggests that neuronal synchrony is not necessarily correlated with visual feature binding.

\subsection{Limitations of Neuronal Synchrony for Visual Consciousness}

Neuronal synchrony seems not to constitute the distinctive mechanism of visual consciousness. Indeed, it has been found that gamma band $(30-60 \mathrm{~Hz})$ synchrony occurs in brains of both conscious and unconscious animals in response to visually presented objects (see Gray and Singer, 1989; Engel et al. 1990). Besides, Lucke and Beach (1998) and Gray (1999) hold that neuronal synchrony correlates tightly with unconscious activity in V1. Moreover, recent experiments reported by Sewards and Sewards (2001) have shown that neuronal synchrony in some anesthetized animals has greater amplitude in the gamma range than in conscious animals under similar conditions. On the other hand, neuronal synchrony has been found in cortical structures not directly related with sensory visual awareness. For example, Sewards and Sewards (2001) have reported neuronal synchrony in the hippocampal formation. They argued that hippocampal formation does not participate in visual awareness based on the fact that lesions in this region do not result in sensory deficits of any kind (ibid. p.492).

In our view, neuronal synchrony does not satisfactorily explain the unity of visual consciousness experience either. The experiencing, conscious mind does not perceive objects or events of a scene as segregated and temporally discrete but rather as unified and continuous in time (Seth et al., 2005; Cosmelli et al, 2007). However, the evidence seems to suggest that neural synchrony explains visual consciousness on the basis of a sequence of "snapshots" or stable states, instead of a unified and continuous process. Rodriguez et al. (1999), who registered EEG phasesynchrony during the perception of highcontrast human faces, observed that emerging stable patterns of phasesynchronous activity in the gamma range were transiently separated from each other by periods of de-synchronization in agreement with the fact that conscious events follow each other with variable delays. Work by Revonsuo et al., (1997) on neuronal synchrony in the gamma range during the fusion of random-dot stereograms seems to confirm this. The authors found that $40-\mathrm{Hz}$ synchronized oscillations were not maintained during the continuous conscious observation of the visual percept, despite the fact that neuronal synchrony seemed to participate in the construction of the visual percept.

Neuronal synchrony would seem thus not be causally related to the emergence of visual consciousness. If neuronal synchrony is held as the putative mechanism of visual consciousness, then it should be able to provide an analytic explanation of the functional organization of the parts involved, specifying how visual consciousness arises. However, the evidence for the role of neuronal synchrony is just correlative: to our knowledge, there is no direct proof that synchrony provokes changes in behavior when it is selectively altered. In fact, there is evidence suggesting that consciousness could be present without neural synchronization. For instance, a remarkable study conducted by Merker (2006) presents detailed evidence that children lacking substantial parts of the cerebral cortex are 
indeed conscious (i.e. they are awake, react emotionally and selectively to events, express pleasure by smiling, etc.).

Finally, we would submit that neuronal synchrony cannot provide an explanation the so-called "hard problem" of qualia either. As discussed elsewhere visual consciousness is private and subjective: it is marked by the existence flow of events that are available and meaningful only for the owner of the brain perceiving them (e.g. Crick and Koch, 2003; Seth et al, 2005). No neuronal correlate has been found to produce a plausible explanation for the private and subjective aspect of consciousness. Of course, neural synchronization does not seem to be the exception.

\section{FINAL CONSIDERATIONS}

If neuronal synchronization does not constitute a mechanism of visual binding as recent evidence suggests, then there is no neurobiological base to support the consciousness by synchrony hypothesis. Certainly this discussion will continue yet, nevertheless, it is unclear what kind of experiments and methodological strategies would be needed to causally relate neuronal synchrony with the "hard problems" of visual consciousness. Moreover, even if we accept the role of neuronal synchrony as causal, some additional problems immediately appear: who coordinates the synchronized activity? Which neuronal assemblies would be "in charge" to interpret that coordination? How could neurons distinguish neuronal inputs as belonging either to local or to large-scale interactions? Would we need an additional neuronal mechanism to specify those distinctions?

\section{REFERENCES}

COSMELLI D, LACHAUX J P and THOMPSON E (2007) Neurodynamical Approaches to Consciousness. In Zelazo P, Moscovitch M, Thompson E (eds.), The Cambridge Handbook of Consciousness. Cambridge University Press

CRICK F C AND KOCH C (1990) Towards a neurobiological theory of consciousness. Seminars in Neuroscience 2: 263-275
CRICK F C AND KOCH C (2003) A framework for consciousness. Nat Neurosci 6: 119-126

ENGEL A, KONIG P, GRAY C, SINGER W (1990) Stimulus-dependent neuronal oscillations in cat visual cortex. Eur J Neurosci 2: 588-606

ENGEL A K, KONIG P, SINGER W (1991a) Direct physiological evidence for scene segmentation by temporal coding. Proc Natl Acad Sci USA 88: 6048-6052

ENGEL A K, FRIES P, KONIG P, BRECHT M, SINGER W (1999) Temporal binding, binocular rivalry, and consciousness. Conscious Cogn 8: 128-151.

ENGEL A K, FRIES P, SINGER W (2001a) Dynamic predictions: oscillations and synchrony in top-down processing. Nat Rev Neurosci 2: 704-716

GRAY C, AND SINGER W (1989) Stimulus specific neuronal oscillations in orientation columns of cat visual cortex. Proc Natl Acad Sci USA 86: 1698-1702

GRAY C M, KONIG P, ENGEL A K, SINGER W (1989) Oscillatory response in cat visual cortex exhibit intercolumnar synchronization which reflects global stimulus properties. Nature 338: $334-337$

GRAY C (1999) The temporal correlation hypothesis of visual feature integration: Still alive and well. Neuron 24: $31-47$

KREITER A K and SINGER W (1996) Stimulus-dependent synchronization of neuronal responses in visual cortex of awake macaque monkey. J Neurosci 16: 2381-2396

LUCK S AND BEACH N (1998) Visual attention and the binding problem: A neurophysiological perspective. In $\mathrm{R}$ Wright (Ed.), Visual attention, 455-478. New York: Oxford University Press

MERKER B (2007) Consciousness without a cerebral cortex: A challenge for neuroscience and medicine. Behav Brain Sci: 30: 63-81

PALANCA B, and DEANGELIS G C, (2005) Does neural synchrony underlie visual feature grouping? Neuron 46: 333-346

REVONSUO A, WILENIUS-EMET M, KUUSELA J, and LEHTO M (1997) The neural generation of a unified illusion in human vision. Neuroreport: $8: 3867-3870$

RODRIGUEZ E, GEORGE N, LACHAUX JP, MARTINERIE J, RENAULT B, \& VARELA, FJ (1999). Perception's shadow: long-distance synchronization of human brain activity. Nature 397: 430-433

ROELFSEMA PR, LAMME VA, SPEKREIJSE H (2004) Synchrony and covariation of firing rates in the primary visual cortex during contour grouping. Nat Neurosci 7: 982-991

ROSKIES AL (1999) The Binding Problem: Special Issue. Neuron 24: 7-9

SETH A K, EDELMAN, DB, BAARS, BJ (2005) Criteria for consciousness in humans and other animals. Conscious Cogn 14: 19-139

SEWARDS TV, and SEWARDS MA (2001) On the correlation between synchronized oscillatory activities and consciousness. Conscious Cogn 10: 485-495

SINGER W (2001) Consciousness and the binding problem. In: P.C. Marijuán (ed.) Cajal and Consciousness: Scientific Approaches to Consciousness on the Centennial of Ramón y Cajal's Textura. Ann N Y Acad Sci 929: 123-146

SHADLEN MN and MOVSHON JA (1999) Synchrony unbound: a critical evaluation of the temporal binding hypothesis. Neuron 24, 67-77

THIELE A and STONER GR (2003) Neuronal synchrony does not correlate with motion coherence in cortical area MT. Nature 421: 366-370

VON DER MALSBURG C (1981) The correlation theory of brain function. MPI Biophysical Chemistry, Internal Report: 81-2 
